\title{
Molecular and toxicological characterization of anti-UV Yeast plasmid compound for skin protection
}

\author{
Raul Cuero ${ }^{1 *}$ and Ibarguen $\mathrm{H}^{2}$ \\ ${ }^{1}$ Former Research Scientist at Prairie View A\&M University, CARC, Member of the Texas A\&M University System, USA \\ ${ }^{2}$ Former Sr. Research Associate at The University of Texas, School of Public Health, USA
}

Key words: Anti-UV, Fibroblast, ATP, Plasmid, LD50

\begin{abstract}
In the present investigation, we characterized an anti-ultraviolet (anti-UV) metabolite from a yeast plasmid, including testing the toxicological effects of the metabolite in vivo. The present work included the study of the effect of the anti UV yeast plasmid metabolite on protecting and maintaining the morphology of skin fibroblast cells when exposed to UV radiation. Moreover, the investigation used ATP as a mediator for protecting skin fibroblasts when exposed to UV radiation. Our results showed that the anti UV metabolite had a molecular weight ranging between 350 -450 Da which was detected by a very intense low mass ion at m/z 118 . Also, small peaks of the $\mathrm{m}+\mathrm{h}$ of valine or other amine were detected, thus indicating a possible induction effect of the identified molecule on anti-UV proteins. The yeast plasmid metabolite also showed high solubility in Dimethyl sulfoxide (DMSO). The metabolite seems to exhibit two protective mechanisms against UV radiation in skin fibroblast cells. These include: a) inducing genes for DNA repair related to anti UV proteins such as heat shock proteins (HSP) and other proteins, and b) providing ATP molecules for mediating and/or ameliorating the effects of UV radiation. These mechanisms help maintain the natural morphology of skin fibroblasts when they are exposed to UV radiation. The anti UV metabolite produced by the yeast plasmid showed a good toxicological safety range when a $\mathrm{LD}_{50}$ test was performed. This research clearly demonstrated the great potential of the anti UV metabolite produced by the yeast plasmid for dermatological studies and/or for protective cosmetic purposes.
\end{abstract}

'This work is part of a Patent Pending'

\section{Introduction}

A fibroblast is a type of cell that is responsible for making the extracellular matrix, including collagen, and actin is one of the main products of fibroblasts in smooth muscle tissue. A specific binding site for actin has been found in fibronectin at the cellular membrane level $[1,2]$. Normal fibroblast cells exhibit an elongated shape, and it is known that they respond to the microtopography of the substratum surface [1]. This is known as 'contact guidance', which is the alignment of the cell in relation to the microstructures of the substratum surface. Contact guidance also influences cell morphology and orientation [2].

Reports from some authors have demonstrated that different chemicals cause both cellular morphological changes and decrease in cell viability [3]. The effect of UV radiation on human skin has been extensively studied, as UV is known to be an environmental factor that causes damage to both animal and plant cells [4]. Other reports have shown the effect of UV on depolymerization of the fibroblast actin protein [5].

The probability of experiencing harmful effects from UV radiation can be decreased through absorption of UV rays before they reach the skin surface. For instance, chemicals in sunscreen are used to absorb a good portion of the UV radiation that would otherwise reach the skin surface, thus protecting the skin from sunburn and/or skin cancer [6].

Numerous substances have been used as UV blockers and/ or sunscreens; however, they are only partially effective or are not biodegradable and can persist in the environment or on the skin longer than necessary [7]. Therefore, there is a great need for new substances that are able to absorb UV radiation while also being biocompatible.

Using synthetic biology to construct an anti-UV yeast plasmid,
Cuero and McKay [8] demonstrated induction of anti-UV proteins to be used for protection of skin cells against UV radiation. This investigation demonstrated induction and construction of anti-UV genes and production of their corresponding proteins such as heatshock proteins (HSP). These researchers were able to achieve protection of skin fibroblast cells as shown by lower percentage of apoptosis of the fibroblast cells when they were treated with this constructed yeast plasmid.

The aim of our investigation was to apply the same anti-UV construct yeast plasmid used previously by Cuero and McKay [8], in order to characterize its metabolites at molecular and toxicological levels, as well as to investigate the skin fibroblast protective mechanism of the plasmid and/or its metabolites. Other parameters such as cellular morphology and ATP production by the yeast plasmid were investigated. Our ultimate aim is to use the results of the investigation for further dermatological research and/or for protective cosmetic purposes.

\section{Materials and methods}

This work was conducted at Prairie View A\&M University - CARC Laboratory, Member of Texas A\&M University System.

\section{Experimental procedure}

Use of anti-UV Yeast plasmid: Cultures of yeast plasmid

Current Corresponding Author: Raul Cuero, PhD, Former Research Scientist at Prairie View A\&M University, CARC, Member of Texas A\&M University System, 17515 Spring Cypress, Suite C-111, Cypress, Texas 77429, USA, E-mail: olimpa@aol.com

Received: July 01, 2016; Accepted: July 11, 2016; Published: July 15, 2016 
previously produced by Cuero and McKay [8] were used to produce the anti-UV compound, which was then used for treating skin fibroblast cells. Additionally, cultures of the yeast plasmid were used for determining ATP concentration. The anti-UV compound was subjected to molecular weight identification as well as different assays including solubility and in vivo toxicity tests.

Production of anti-UV Yeast compound: This was carried out by following procedures by Cuero and McKay [8]. Fresh liquid yeast cultures were grown for $24-48$ hour at $28-30^{\circ} \mathrm{C}$ under shaking conditions (300 RPM). Aliquots from this culture at 0.07 O.D. (determined at 550-600 nm) were taken to make triplicates cultures of $250 \mathrm{~mL}$ each. Culture $\mathrm{pH}$ was adjusted to $4-4.5$. These yeast culture solutions were pooled into $1 \mathrm{~L}$ sterile glass bowls and were incubated under 12 hour alternating regime (on-off) of shortwave UV radiation $(254 \mathrm{~nm}) .48$ hour yeast cultures were used in the present investigation.

\section{Molecular characterization}

\section{Molecular weight determination}

These tests were carried out by CEETOX Lab (Kalamazoo, Michigan, USA), according to standard procedures A Fully Automated Kinetic Solubility Screen in 384-well Plate Format using Nephelometry $[9,10]$.

Initially, yeast pellet samples were diluted into water, water plus acetonitrile, and water with $0.1 \%$ formic acid plus acetonitrile; a drop of hydrochloric acid was also added to the solution. This solution was used for further analysis, in order to determine the molecular weight of the yeast anti-UV compound.

The samples were separated by HPLC (Waters Alliance 2795) using a Phenomenex Synergi Hydro-RP8 80A $150 \times 2.0 \mathrm{~mm}$ column with 4 micron particle packing $(00 \mathrm{~F}-4375-\mathrm{BO})$ at a flow rate of $1 \mathrm{~mL} / \mathrm{min}$ and with the temperature maintained at $50^{\circ} \mathrm{C}$. Solvent A was water plus $0.1 \%$ formic acid. Solvent B was acetonitrile with $0.07 \%$ formic acid. The gradient timetable was determined.

Sample solutions were infused into the mass spectrometer via the electrospray (ESI) probe. After preliminary settings were found, each sample was infused and full scan spectra acquired. The mass spectrometer was a Waters Quattro model. Two sample solutions were run: 1) solution from non-UV treated yeast (NUVY1 = control), and 2) solution from UV-treated yeast (UVY2).

\section{Solubility test of the anti-UV compound}

The solubility test was performed at the CEETOX Lab (Kalamazoo, Michigan, USA) following standard protocols [9-11]. The test compounds were prepared in DMSO and the appropriate amounts were then added to complete medium containing $10 \%$ bovine serum and $10 \%$ calf serum at $37^{\circ} \mathrm{C}$. The samples were evaluated using a light scattering technique using a Nepheloskan Nephelometer (Thermo Fisher Scientific). A reading that was greater than or equal to 3 times background was considered the limit of solubility.

\section{ATP analysis of Yeast plasmid culture}

This was carried out using a combination of extraction and quantitation methods, following Promega (Wisconsin, USA) and Invitrogen (Massachusetts, USA) assay protocols, using a yeast cell culture that had been used to induce synthesis of the anti-UV compound.

First, ATP extraction was performed following a Promega protocol.
Trichloro acetic acid was mixed with a cell sample $(1 \mathrm{~mL})$, followed by centrifugation and addition of Tris buffer to the supernatant. a ten $\mu \mathrm{L}$ aliquot was removed and saved for the quantitative analysis of ATP.

The ATP quantitation was performed using the Invitrogen ATP Determination Kit (A22066), which is a quantitative bioluminescence assay that uses a $10 \mathrm{~mL}$ standard reaction solution. ATP quantitation was carried out following a standard curve based on serial dilution of ATP standard reaction solution. Aliquots of this ATP standard solution were placed in a Luminometer (I-20/20 Promega) to determine the luminescence. Aliquots of experimental samples were exposed to the Luminometer to read their luminescence and compared to the level of luminescence of the serial dilution of the ATP standard curve. Results are expressed as relative luminescence unit (RLU)

\section{Toxicological characterization}

\section{In vivo animal toxicity test}

An in vivo assay in mice was also used to demonstrate the safety of the anti-UV compound in a single dose tolerance study.

The in vivo animal assay was carried out by ViviSource Laboratory (Waltham, MA, USA). The test was done following regulatory compliance regarding animal studies. The study was also performed in compliance with the Standard Operating Procedures of ViviSource Laboratories Inc.. Animal care and procedures in this protocol were in compliance with the Animal Welfare Act [12,13], the Guide for the Care and Use of Laboratory Animals, and the Office of Laboratory Animal Welfare [12,13].

Male and female mice (strain CD-1) were used as the test system. Animals used weighed 18-22 grams and were provided standard housing, rodent diet (PharmaServ lab diet 5001), and RO water. The feed was analyzed by the supplier detailing nutritional information and levels of specified contaminants. The water supply was monitored by the City of Waltham.

The anti-UV test compound was stored protected from light and desiccated in a refrigerator set to maintain approximately $4^{\circ} \mathrm{C}$ until use. DMSO and 95\% sterile deionized water were used as vehicle to deliver the anti-UV compound to the mice.

The anti-UV test material was prepared just prior to the animals being dosed. Mice were randomized into groups, identified individually, and weighed. Mice were dosed via tail vein IV in a slow bolus push of $10 \mathrm{~mL} / \mathrm{kg}$. Three male and three female mice received dosing vehicle only. Animals were observed for up to 3 hours post dosing for signs of abnormal behavior and recorded. Daily health observations were performed for 7 days post dose. Body weights of the animals were also performed. On day 8 , mice were euthanized and a gross necropsy was performed to look at any abnormalities in the liver, heart, kidneys, visceral fat, and brain. All observations were recorded.

\section{Data analysis of the in vivo test}

A Probit analysis was performed using GraphPad Prism version 4.03. The dose effecting $50 \%$ death (lethal dose $50 \%$ or $\mathrm{LD}_{50}$ ), was reported along with $95 \%$ confidence intervals (C. I.).

\section{In vitro test of anti-UV compound in skin fibroblast cells (ATCC 2122-CRL)}

Cellular morphological change was use as a parameter to determine the effect of the anti-UV compound on fibroblast cell culture. After 24 hours of incubation, fibroblast cells were harvested by trypsinization 
according to the supplier's protocols and subjected to microscopic analysis. Several fields were observed under 40X magnification using a compound Zeith microscope with an attached camera.

The ability of anti-UV extract from yeast cells to protect skin fibroblast cells against UV radiation was tested in vitro. Skin fibroblast cells were used as a model for human skin and were maintained in culture media for propagation and renewal following ATCC recommendations. (ATCC-formulated Eagle's Minimum Essential Medium, Catalog No. 30-2003). Fetal bovine serum was added to the medium to a final concentration of $10 \%$ as a supplement to the medium.

\section{Maintenance and sub-culturing of fibroblast cells for testing the anti-UV extracts}

Fibroblast cells were maintained in culture according to ATCC recommendations. For sub-culturing, old medium was removed followed by rinsing the flask containing fibroblast cells with $0.25 \%$ trypsin and $0.03 \%$ EDTA solution, followed by removing the trypsin and incubation for 2 to 5 minutes at room temperature $\left(26^{\circ} \mathrm{C}-28^{\circ} \mathrm{C}\right)$. Then, fresh supplemented medium was added into the flask and fibroblast cells were subculture in a ratio of 1:3 according to ATCC instructions.

\section{Treatment}

Extracts from 48 hours yeast cell cultures were prepared as described above and were applied to fibroblast cultures and mixed thoroughly. $1 \mathrm{~mL}$ of yeast extract was used to treat $100 \mathrm{~mL}$ of fibroblast culture. Fibroblast cells were diluted to a concentration of $2 \times 10^{6}$ cells $/ \mathrm{mL}$ for further use. Each experiment was performed in triplicate in canted culture flask $\left(75 \mathrm{~cm}^{2}\right.$; Falcon 353135), for which the top part had been removed aseptically in a microbiological laminar flow cabinet in order to aloud direct penetration of the UV radiation to the fibroblast culture before incubation. Transferring of the fibroblast cell culture into the canted culture flask was carried out aseptically inside of the UV-CO, incubator, which was pretreated for 24 hours in order to provide and ensure aseptic conditions for incubation of the fibroblast cultures.

This cell mixture was then exposed to UV radiation for 24 hours to simulate the natural exposure of the skin to UV radiation. Each time, the experiment includes fibroblast cell cultures treated and untreated with the extract of transfected S. cerevisiae.

Some treated fibroblast were not irradiated as a control. Further, untreated fibroblast cells with and without irradiation were incubated for 24 hours at $37^{\circ} \mathrm{C}$ and $5 \% \mathrm{CO}_{2}$ while being exposed to $254 \mathrm{~nm} \mathrm{UV}$ radiation. Control non-treated fibroblast cells were incubated under same conditions $\left(24\right.$ hours at $37^{\circ} \mathrm{C}$ and $5 \% \mathrm{CO}_{2}$ while being exposed to $254 \mathrm{~nm}$ UV radiation).

\section{Results}

\section{Determination of molecular weight of the anti-UV yeast compound}

The anti-UV compound was identified as a metabolite ranging a molecular weight between $350-450 \mathrm{Da}$ which was detected by a very intense low mass ion at $\mathrm{m} / \mathrm{z} 118$. Also, small peaks of the $\mathrm{m}+\mathrm{h}$ of valine or oanther amine were detected (Figure 1).

\section{Determination of solubility of the anti-UV yeast compound}

This compound showed a good solubility of $0.015 \mathrm{mg} / \mathrm{mL}$ in DMSO at 24 hours and 72 hours in a solubility test.

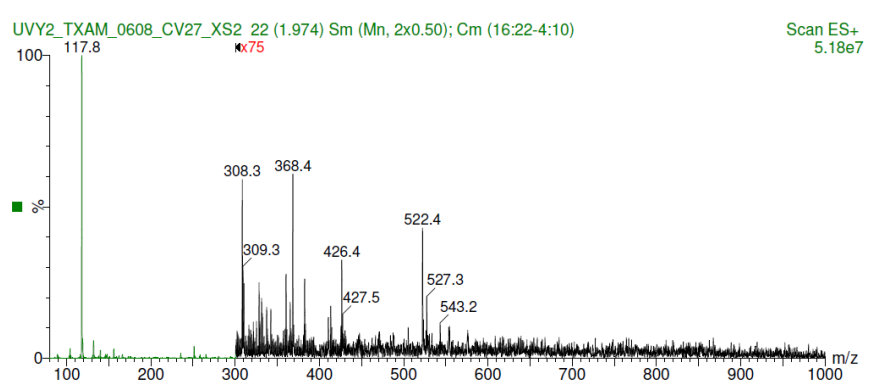

Figure 1. Shows the molecular weight range between $350-450 \mathrm{Da}$ including a very intense low mass ion at $\mathrm{m} / \mathrm{z} 118$.

Table 1. ATP production in yeast plasmid cells before and after UV radiation, as compare to non-transformed yeast cells.

\begin{tabular}{|l|c|}
\hline Treatments & $\begin{array}{c}\text { ATP (RLU) } \\
\text { Mean +/- SD }\end{array}$ \\
\hline Yeast plasmid cell + no UV & $115 \pm 20$ \\
\hline Yeast plasmid cell + UV & $93 \pm 9$ \\
\hline non-transformed yeast cell + no UV (Control) & $102 \pm 15$ \\
\hline non-transformed yeast cell + UV (Control) & $87.9 \pm 12.1$ \\
\hline
\end{tabular}

\section{ATP determination in yeast plasmid cells}

Table 1 shows that the ATP concentration expressed as relative luminescence units (RLU) of both the yeast plasmid-containing and non-transformed yeast culture decrease when exposed to UV radiation. However, the ATP concentration was higher on the irradiated yeastplasmid-containing culture than on the non-transformed yeast cells (control).

\section{In vivo test}

Table 2 shows that the safer dose for the anti-UV compound was less than $20 \mathrm{mg} / \mathrm{kg}$ according to the predicted value of $\mathrm{LD}_{50}$. However, a no-effect dose was equivalent to $1-2 \mathrm{mg} / \mathrm{kg}$.

\section{Effect of the anti UV Yeast plasmid on protection of skin fibroblast cells}

The original typical elongated shape and size $20-40 \mu \mathrm{M}$ of the fibroblast cells treated with the anti-UV plasmid and exposed to UV radiation was maintained similarly to fibroblast control cells that were not treated nor exposed to UV radiation (Figures 2 and 4). On the contrary, fibroblast cells exposed to UV radiation without antiUV protection treatment showed an atypical spherical shape and a reduction in size; further, some of these cells showed pyknotic nuclei or necrotic or to be undergoing apoptosis (Figure 3).

\section{Discussion}

The molecular weight of the anti-UV compound is in the range of 350-450 Da, which was also close to a peak of valine and/or other amine groups (Figure 1). It is possible the identified molecule is inducing the production of proteins including those with anti-UV properties and/or DNA repair properties. Cuero and McKay 2013 [8] found expression of upregulated proteins such as heat shock proteins (HSP), hexokinase and alcohol dehydrogenase (ADH) when they were used as parts of the yeast plasmid that demonstrated anti-UV activity. This yeast plasmid is the same that was used in the present investigation, thus corroborating the present results.

Treatment of yeast cells with chemical or physical agents (such as 
Table 2. In vivo toxicological test of the anti-UV compound, using an $\mathrm{LD}_{50}$ assay in mice at 24 and 72 hours.

\begin{tabular}{|c|c|c|c|c|c|c|}
\hline \begin{tabular}{|l|} 
Compound \\
Time of \\
Analysis \\
\end{tabular} & $\begin{array}{l}\text { Cell Number } \mathrm{TC}_{50} \\
(\mathrm{mg} / \mathrm{mL})\end{array}$ & $\begin{array}{c}\text { MemTox } \\
\text { TC }_{50} \\
(\mathrm{mg} / \mathbf{m L})\end{array}$ & $\begin{array}{c}\text { ATP } \\
\text { TC }_{50} \\
(\mathrm{mg} / \mathbf{m L})\end{array}$ & $\begin{array}{c}\text { Predicted } \\
\mathrm{C}_{\text {tox }} \\
(\mathrm{mg} / \mathrm{mL})\end{array}$ & $\begin{array}{c}\text { Predicted } \\
\text { LD }_{50} \\
(\mathrm{mg} / \mathrm{kg})\end{array}$ & $\begin{array}{c}\text { Predicted } \\
\text { No Effect Dose } \\
(\mathrm{mg} / \mathrm{kg})\end{array}$ \\
\hline \begin{tabular}{|l} 
Anti-UV \\
H4IIE 24HR
\end{tabular} & $>1$ & 0.57 & 0.14 & 0.266 & $\begin{array}{l}20-50 \\
\mathrm{mg} / \mathrm{Kg}\end{array}$ & $\begin{array}{c}1-2 \\
\mathrm{mg} / \mathrm{Kg}\end{array}$ \\
\hline \begin{tabular}{|l} 
Anti-UV \\
H4IIE 72HR
\end{tabular} & 0.04 & 0.02 & 0.01 & NA & NA & NA \\
\hline
\end{tabular}

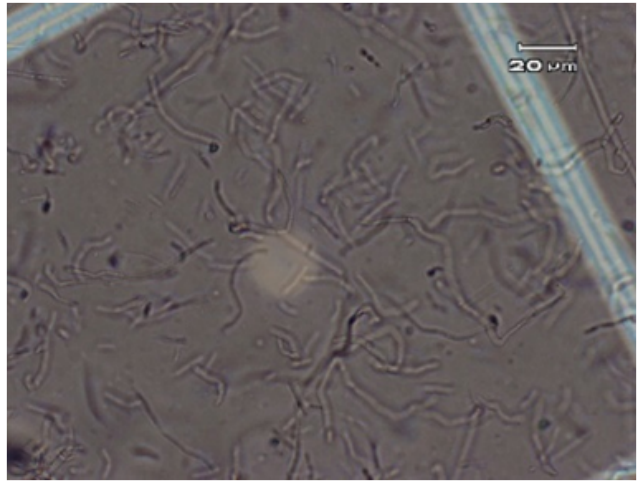

Figure 2. Control, normal elongated fibroblast alone (no anti-UV treatment and no exposure to UV radiation)

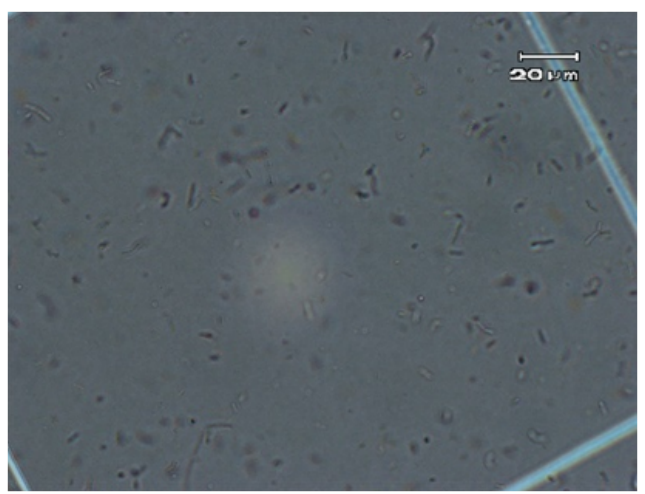

Figure 3. Spherical shape of fibroblast cells without anti-UV compound protection after exposure to UV radiation.

UV) may lead to a series of molecular changes within the cell. These changes may affect multiple cell signal transduction pathways and subsequently cause following outcomes:

1. Upregulation or down regulation of cellular proteins at cytoplasmic level.

2. Differential expression of functional proteins in response to activation/ deactivation of genes expression.

3. Regulation of gene expression by various transcriptional factors.

Also, mammalian cells respond to a variety of stimuli behave differently at the cellular and molecular level, for instance in the fibroblast growth factor(s) signaling pathways [15]. At the cellular level, secreted FGFs regulate fundamental cellular processes that include positive and negative regulation of proliferation, survival, migration, differentiation, and metabolism [16-18].

The identified anti-UV compound also showed good solubility, $0.015 \mathrm{mg} / \mathrm{mL}$ in DMSO, at 24 hours and 72 hours in a solubility test.

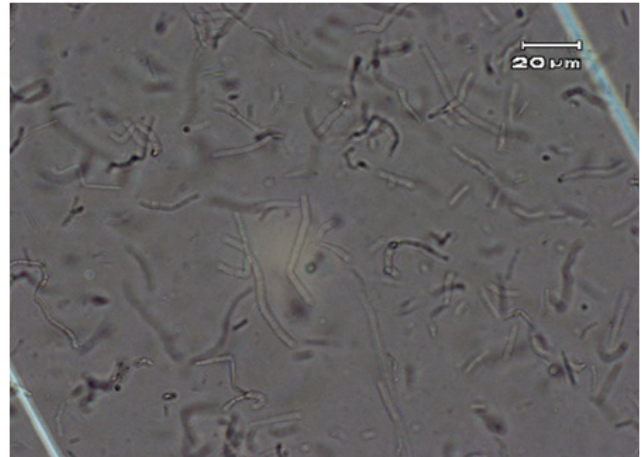

Figure 4. Normal elongated fibroblast treated cells with the anti-UV compound and exposed to UV radiation.

This suggests that this compound can be readily dissolved in any polar organic solvent as well as water, and it could either be absorbed by the skin or it might have an external protective effect on the skin cells. Thus, it may have applications in UV skin protection products.

The positive results of the present investigation warrant further studies on the bioavailability as well as basic pharmacokinetic analyses of the anti-UV compound.

The present results also suggest that ATP has a role in mediating the effects of UV radiation on skin fibroblast cells (Table 1).

Actin is a primary component of the cytoskeleton in skin fibroblast cells and others [19] it regulates the cell shape in response to physiological changes caused by chemicals and physical agents including UV radiation. A previous report has demonstrated that the depolymerization and polymerization of actin is regulated by ATP hydrolysis [20].

Perhaps a similar mechanism is involved in the biological system used in our investigation where, ATP concentration varied between yeast plasmid cells and non-transformed yeast cell culture. Table 1 shows evidence of the effect of UV radiation on ATP concentration. Thus, yeast plasmid cultures that showed protection of the skin fibroblast against UV radiation, showed higher ATP concentration as compared to the non-transformed yeast cells. However, ATP concentrations decreased in both yeast plasmid and non-transformed yeast cultures when they were exposed to UV radiation. Perhaps this corroborates the mediating role of the ATP on UV radiation in relation to the protection of skin fibroblasts. This suggests that the protection of skin fibroblasts can be maximized by increasing the number of cells in the yeast plasmid cultures .

Perhaps the mechanism for protection of the morphological architecture of the fibroblast cells when using the anti UV compound could be explained by the interaction between ATP and UV radiation. For instance, a decrease in concentration of ATP due to its hydrolysis induced by the UV exposure would imply use of the ATP molecules to mediate the effect of the UV radiation, thus helping to protect and maintaining the normal morphology of the fibroblast. The maintenance 
Figure : Fibroblast + Anti-UV Compound.
D

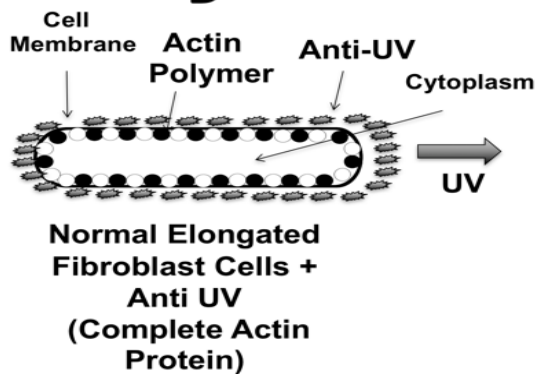

E

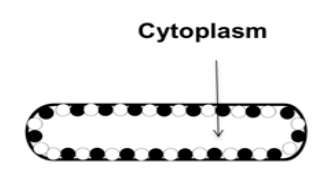

Normal Elongated

Fibroblast Cells

(Complete Actin Protein)
Figure 5. Schematic of how fibroblast cells exposed to UV radiation without anti UV yeast plasmid may become spherical. According to this hypothesis, actin becomes depolymerized due to the effect of the UV radiation; actin may even be fragmented from the membrane (Figure 5A, 5B, 5C). On the contrary, when fibroblast cell culture is exposed to UV radiation but protected with the anti-UV yeast plasmid compound, the cells therein maintain their elongated natural shape. This could be due to the effect of the anti-UV yeast plasmid metabolite in preventing monomerization of the actin of the fibroblast cytoskeletin; in this instance, the actin appears to be completely integrated to the cell membrane (Figure 5D, 5E).

of the elongated morphology of the fibroblast is an indication of cell viability. This maintenance of fibroblast morphology correlates with a low percentage of apoptosis in fibroblast cells that were treated with the same type of yeast plasmid [8]. Other authors Lake et al. [21] have demonstrated in normal human fibroblast cells the importance of ATP hydrolysis to maintain the healthy structure of chromatin bonded to Cockayne syndrome complementation group B protein (CSB), which is necessary for repairing DNA lesions after UV irradiation [8]. This supports the present results, since in the yeast plasmid that we used contains genes involved in DNA repair.

Figure 5 illustrates a hypothetical mechanism involving the effect of the anti-UV yeast plasmid molecule on protecting the skin fibroblast cells. The diagram shows how the fibroblast cells exposed to UV radiation without the protection of the anti-UV yeast plasmid molecule develop a spherical shape (Figure 5A-5C), whereas fibroblast cells exposed to UV radiation but treated with the anti-UV yeast plasmid molecule maintained their natural elongated shape (Figure 5D and 5E). Perhaps, the anti-UV yeast plasmid molecule prevents the monomerization of the actin protein when the fibroblast cells are exposed to UV radiation. Thus, the fibroblast cells maintained the expected shape. Different authors, using different biological systems studied the effect of the different types of physical radiation, including heat and UV on the integrity of the actin polymer [22,23].

The anti-UV yeast plasmid compound not only shows effectiveness on protecting fibroblast cells, but also demonstrates a low level of toxicity with respect to the $\mathrm{LD}_{50}(1-2 \mathrm{mg} / \mathrm{kg})$. However, it is still important to carryout bioavailability and some basic pharmacokinetic endpoint studies, ideally in a single or repeat dose 3 or 4 day rat study [24]. Two routes should be used, the intended route (oral) and intravenous bolus in order to collect pharmacokinetic parameters (i.e., blood, urine, feces samples). These would allow a determination of the half-life, metabolism or metabolic stability, and general tolerance by animals [24]. Alternatively, the bioavailability $(\% \mathrm{~F})$ can be estimated by software designed for this purpose, since we already know the molecular weight and the solubility of the main compound in the anti UV yeast plasmid.

\section{Conclusions}

1. The anti-UV yeast plasmid contains genetic material responsible for producing a molecule that seems to exhibit two protective mechanisms on skin fibroblast cells against UV radiation. The two mechanisms include: a) inducing genes for DNA repair such as HSP and other proteins, and b) providing ATP for mediating and/or ameliorating the effects of UV radiation. Therefore, this molecule helps to maintain the natural morphology of skin fibroblasts when they are exposed to UV radiation.

2. The present research has demonstrated the importance of ATP as a mediator for protecting skin fibroblasts when exposed to UV radiation.

3. The anti-UV molecule produced by the yeast plasmid showed good molecular and toxicological characteristics, making the molecule ideal for skin protection. The molecule should thus be used for dermatological studies and/or for protective cosmetic purposes.

\section{Acknowledgments}

This research was supported by a grant from the NASA Lyndon B. Johnson Space Center-Houston (NASA Grant \# NAG-1241), and from CARC-Prairie View A\&M University-Evans Allen Fund. The Main Author thanks the late Dr. David S. McKay, former Scientist from NASA JSC-Houston for his valuable technical contribution, Dr. Eustace Duffus for his effective laboratory technical assistance, and to Dr. Larry Villanueva for reviewing the manuscript.

\section{References}

1. McKeown-Longo PJ, Mosher DF (1983) Binding of plasma fibronectin to cell layers of human skin fibroblasts. J Cell Biol 97: 466-472.[Crossref]

2. Eisenbarth E, Meyle J, Nachtigall W, Breme J (1996) Influence of the surface structure of titanium materials on the adhesion of fibroblasts. Biomaterials 17: 1399-1403. [Crossref]

3. CamesanoTA, NatanMJ, LoganBE (2000) Observation of Changes in Bacterial Cell Morphology Using Tapping Mode Atomic Force Microscopy. Langmuir16.

4. Kovács E, Keresztes A (2002) Effect of gamma and UV-B/C radiation on plant cells. Micron 33: 199-210.[Crossref]

5. Grzanka D, Domaniewski J, Grzanka A, Zuryn A (2006) Ultraviolet radiation (UV) induces reorganization of actin cytoskeleton in CHOAA8 cells. Neoplasma 53: 328332.[Crossref]

6. KimbroughDR (1997) The photochemistry of sunscreens. Journal of Chemical Education 74: 51.

7. CockellCS, Knowland J (1999) Ultraviolet radiation screening compounds. Biological Reviews of the Cambridge Philosophical Society 74: 311-345.

8. Cuero R, McKay DS (2013) Induction and construct UV protective yeast plasmid. $J$ Biotechnol 166: 76-83.[Crossref]

9. Bevan CD, Lloyd RS (2000) A high-throughput screening method for the determination of aqueous drug solubility using laser nephelometry in microtiter plates. Anal Chem 72: 1781-1787.[Crossref]

10. TestaB, Van DE, Waterbeemd H, Folkers G, Guys R (2001) Pharmacokinetic Optimization in Drug Research, Verlag Helvetica Chimica Acta, Zürich: 257-73

11. Agilent Technologies (2001) Printed in the U.S.A. February. 5988-2045EN.

12. Rozemond H (1986) Laboratory animal protection: the European Convention and the Dutch Act. Vet $Q$ 8: 346-349.[Crossref]

13. International Guiding Principles for Biomedical Research Involving Animals (2012) Council for International Organization of Medical Sciences and The International Council for Laboratory Animal Science.

14. KumarV, Robbins S (2007) Robbins Basic Pathology.Saunders/ElsevierPhiladelphia.

15. Ornitz DM, Itoh N (2015) The Fibroblast Growth Factor signaling pathway. Wiley 
Interdiscip Rev Dev Biol 4: 215-266.[Crossref]

16. Yamanaka Y, Lanner F, Rossant J (2010) FGF signal-dependent segregation of primitive endoderm and epiblast in the mouse blastocyst. Development 137: 715-724.[Crossref]

17. Krawchuk D, Honma-Yamanaka N, Anani S, Yamanaka Y (2013) FGF4 is a limiting factor controlling the proportions of primitive endoderm and epiblast in the ICM of the mouse blastocyst. Dev Biol 384: 65-71.[Crossref]

18. Müller AK, Meyer M, Werner S (2012) The roles of receptor tyrosine kinases and their ligands in the wound repair process. Semin Cell Dev Biol 23: 963-970.[Crossref]

19. Vindin H, Gunning P (2013) Cytoskeletal Tropomyosins: choreographers of actin filament functional diversity. Journal of Muscle Research and Cell Motility 34: 261-74.

20. Otterbein LR, Graceffa P, Dominguez R (2001) The crystal structure of uncomplexed actin in the ADP state. Science 293: 708-711.[Crossref]
21. Robert JLake,Anastasia Geyko,Girish Hemashettar,Yu Zhao and Hua-Ying Fan (2010) UV-Induced Association of the CSB Remodeling Protein with Chromatin Requires ATP-Dependent Relief of N-Terminal Autorepression. Molecular Cell 37: 235-246.

22. XiangL, RensingL (1999) Changes in cell morphology and actin organization during heat shot in Dictyostelium discoideum: does HSP70 play a role in acquired thermotolerance?FEMS Microbiol Lett178:95-107

23. SommarugaR, Oberleiter A, Psenner R (1996)Effect of UV Radiation on the Bacterivory of Heterotrophic Nanofagellate. Applied and Environmental Microbiology 62: 4395-4400.

24. Lipnick RL, Cotruvo JA, Hill RN, Bruce RD, Stitzel KA, et al. (1995) Comparison of the up-and-down, conventional LD50, and fixed-dose acute toxicity procedures. Food Chem Toxicol 33: 223-231.[Crossref]

Copyright: O2016 Cuero R. This is an open-access article distributed under the terms of the Creative Commons Attribution License, which permits unrestricted use, distribution, and reproduction in any medium, provided the original author and source are credited. 